\title{
The role of isotonic strength training for lateral epicondylitis
}

\author{
Rabea Begum ${ }^{1 *}$ and Mohammad Habibur Rahman ${ }^{2}$ \\ ${ }^{1}$ Physiotherapy Department, Centre for the Rehabilitation of the Paralyzed, Bangladesh \\ ${ }^{2}$ The School of Science and Technology, Bangladesh Open University, Bangladesh
}

\begin{abstract}
Background: Lateral epicondylitis is the painful musculoskeletal condition that is caused by overuse injury. This injury not only occur in tennis player but also happen in other sports like racquetball, squash, weight lifting and jobs involving highly repetitive movement. The patients complain of pain and tenderness over the lateral epicondyle of the humerus, weakness at grip and loss of function. This condition is confirmed by cozen's test, mill's test. Research on physiotherapy still not proven the superiority of any specific approach. Isotonic strength training is one of component of rehabilitation. The muscle length is changing by this strength training (concentric and eccentric).
\end{abstract}

Aim: The aim of this study is to find out the role of isotonic strength training for patients with lateral epicondylitis.

Discussion: The isotonic exercise reduces the chance of recurrence and help to athlete for returning to play as early as possible. The concentric exercise and eccentric exercise are not differed separately for the outcome of pain, strength and function in lateral epicondylitis patients Isolated eccentric exercise is not useful whereas effective with combined approaches in stretching and isometric strength training. Conclusion: The scientific evidence supports the effectiveness of isotonic strength training especially eccentric exercise for patients with lateral epicondylitis.

\section{Introduction}

Lateral epicondylitis is commonly known as tennis elbow. It is referred to the inflammation of common wrist extensor group of muscles especially in extensor carpi radialis brevis. Histological examination showed that there is deficiency of acute inflammation cells in lateral epicondylitis $[1,2]$.

Tennis elbow is not an uncommon condition in upper extremity. The prevalence of tennis elbow in $3.8 \%$ in Japan and female are more prevalent and affected population in between 50 years to 59 years of age [3]. The prevalence of lateral epicondylitis in the general population is approximately $1.0-1.3 \%$ in men and $1.1-4.0 \%$ in women [4].

A descriptive study was done by Sanders, et al. [5] and reported that the incidence of tennis elbow is 3.3 to 3.5 per 1000 population. The peak age between 40 to 49 years in their productive ages. The recurrence rate was $8.5 \%$.

It is an overuse painful tendinopathy that affect elbow joint and radio-humeral joint. The characteristic of tennis elbow comprises pain over lateral epicondyle, pain during shaking of hands, poor grip strength. The occupation, sports and domestic activities that are related with repetitive wrist extension there are high risk for developing tennis elbow.

The athletes who play tennis, baseball, volleyball, golf have risk for developing tennis elbow. The computer keyboard or mouse use increases the risk. This condition is not only found among tennis player but also common in persons who perform repetitive upper body activities such as carpenters, musicians and computer programmers. The activity of extensor carpi radialis brevis are reduced and also found upper limb muscle weakness due to disuse and fear of avoidance [6].
The common cause of tennis elbow found in tennis. It is predictable that $50 \%$ tennis elbow occur in tennis players. The significant risk factors have been identified include improper technique and the size and weight of the racquet. The pathophysiology tennis elbow involves inflammatory processes of the radial humeral bursa of fluid-filled sac and nearby ligaments due to microscopic tearing with formation of scar tissue at the area of origin of the extensor carpi radialis brevis tendon [7].

An epidemiological study by Kachanathu et al. [8] was done in India on tennis player. Total 350 professional tennis players were engaged in this study. After sampling 256 participants were selected. The male participants were more than female. The elbow joints were the most frequent joint (29\%) than other joint like shoulder, wrist, hip, knee, ankle, calf. The backhand was the aggravating hit for this elbow injury. The incidence of injury in less than 16 years was more than aged in 26 years. They also found that those athletes had done warm up the incidence of injury was decreased. The athlete who did not perform any warm up session they developed maximum prevalence of injury.

Tennis elbow is the common disorder of the elbow. The extrinsic and intrinsic factors are responsible to develop tennis elbow. There was found association between repetitive movements of the arms and forceful activities for developing tennis elbow [4]. The intrinsic

*Correspondence to: Mst. Rabea Begum, MPT, MPH (NCD), Senior Clinical Physiotherapist, Physiotherapy Department, Centre for the Rehabilitation of the Paralysed (CRP), Savar, Dhaka-1343, Bangladesh, E-mail: physio_miilee@yahoo.com

Key words: lateral epicondylitis, isotonic strength training, eccentric strength training, concentric strength training

Received: April 25, 2019; Accepted: May 08, 2018; Published: May 13, 2018 
factors alteration of joint arthrokinematics, muscular imbalances or muscular weakness in the upper extremity. These factors revealed to microtrauma in involved tissues. A study by Lucado et al. [9] found that poor muscular strength of shoulder, elbow and wrist extensor are responsible for cause- effect relationship for developing tennis elbow. It was a cross sectional study. In result the data also showed that there were significant differences in muscle strength ratios for the scapular musculature, shoulder rotators, and wrist musculature of the female tennis players who had symptoms of lateral epicondylitis.

Lateral epicondylitis is examined and confirmed by Cozen's test. It is also known as the resisted wrist extension test. The aim of this test is to check for tennis elbow. The procedure is the elbow is stabilized in $90^{\circ}$ flexion. The therapist palpates the lateral epicondyle and the other hand positions the patient's hand into radial deviation and forearm pronation. Then the patient is asked to resist wrist extension. The test is positive if the patient experiences a sharp, sudden, severe pain over the lateral epicondyle. The sensitivity and specificity have not till determined.

Longitudinal studies have shown that this injury takes time to heal from 6 months to 12 months or 1 year. If partial rupture is occurred with 3 to 4 weeks tissue is going to heal [10]. If surgery is needed, then 12 weeks require for recovery. The symptoms of tennis elbow resolve in between 12 to 18 months without treatment [11].

In management of lateral epicondylitis non-operative medical treatment is effective. Physiotherapy is one of core component to rehabilitate in patients with lateral epicondylitis. The rehabilitation phases have some component: muscle conditioning, flexibility, proprioception and others. The isotonic strength training is a part of muscle conditioning. The aim is to maintain the length of muscle fibers. Literature found the effectiveness of isotonic strength training (concentric and eccentric) in reduction of pain, improvement of function and strength for lateral epicondylitis patients.

On the date of 21th September 2017 I went to Tennis Federation, Ramna, Dhaka. After reaching receptionist referred me to the administrative officer, Mahmud Alam. This person introduced me with the coach of tennis so that I can find out the injured player. I found two injured players on that time. $1^{\text {st }}$ player aged 15 years, left hand dominant, continue education at class VII. During playing he had been suffering pain at his elbow from 1 year ago. After that he was managed this pain by pain killers. Still now he is suffering his pain and score of pain is in VAS 5/10. Though there is no physiotherapist athlete has no knowledge about this pain management by physiotherapist [12] The $2^{\text {nd }}$ tennis player is 17 years old and he also continues education in class VII. He is playing tennis for more than 4 years. He felt elbow pain for 6 months. Now there is no pain. This player managed his pain by some stretching exercise and maintained proper cool down exercise according to player statement. He could not receive any drugs to manage his pain [12]. The coach taught this type of exercise. The coach said that if any tournament is coming up then recruit a physiotherapist for their pain management.

\section{Justification of the study}

Lateral epicondylitis is more common in tennis player as well as who are doing repetitive use of wrist. The sports physiotherapists play a vital role in acute pain management and in rehabilitation. Lateral epicondylitis or tennis elbow can impact on social and professional life as $50 \%$ of tennis player suffered this condition. It can also influence on the performance of athlete. Conservatively and surgically tennis elbow is managed. The physiotherapy management is universal technique for tennis elbow patients. The aim of different approaches are used for reducing pain, improving range of movement and grip strength as an intervention of lateral epicondylitis. These types of are beneficial for athletic performance as well as return to play as early. The isotonic strength training is one of the components of physiotherapy that helpful to decrease pain, progress strength and functional ability. The aim of this study is to describe the role of isotonic strength training in patients with lateral epicondylitis.

To date no universal treatment, exist but some principles of treatment should be taken in to consideration. The physiotherapy treatment of lateral epicondylitis is designed to the management of pain, preservation of movement, improvement in grip strength and endurance, return to normal function and control of further clinical deterioration. The aim of this study was to find out the evidence based manual therapy for lateral epicondylitis or tennis elbow.

A systematic review of randomized trials by Cullinane et al. [13] on eccentric exercise for lateral epicondylitis. The outcome measures were on pain, function and strength. Total 12 studies were selected. Among them 3 studies were high quality, 7 were medium and two studies were in low quality. One study found that isolated eccentric exercise is not benefited for improving pain in patients with lateral epicondylitis when compared with others program. One medium quality study found that eccentric exercise with other therapy is not effective than Cyriax therapy program. The Cyriax uses the deep transverse friction massage in combination with Mill's manipulation. In this randomized clinical trial author recommended that Cyriax physiotherapy is a superior treatment approach than eccentric exercise. Majority of studies in this systematic review showed improvement in pain, function and grip strength when eccentric exercise was added with other therapies. There was no adverse effect found from the prescription of eccentric exercise. Finally, this systematic review supports for using eccentric exercise with combination of other therapies.

\section{Initial management of lateral epicondylitis}

Tennis elbow or lateral epicondylitis is one kind of tendinitis that causes pain and tenderness at elbow and arm. In initial management use of elbow strap is important to protect the injured tendon from further strain. Icing the elbow is also clinically significant to reduce pain and swelling. Mac Auley [14] suggested that the application of ice through a wet towel for 10 minutes is effective for muscle injury. The target is for reducing the temperature in $10-15^{\circ} \mathrm{C}$ that prevents further injury also. But experts recommend that icing for 20 to 30 minutes every 3 to 4 hours for 2 to 3 days or until the pain has gone.

\section{Rehabilitation of lateral epicondylitis}

Tennis elbow is degeneration of the tendons that attach to the lateral epicondyle that causes pain and tenderness at elbow. The aim of rehabilitation is to enable the athlete to return to sports with full function in the shortest possible time. The rehabilitation program consists of muscle conditioning, flexibility, proprioception, function exercise, sports skills, correction of abnormal biomechanics and maintenance of cardiovascular fitness.

Eccentric strength training is the rehabilitative component of rehabilitation in patient with lateral epicondylitis. The eccentric exercises can effectively lengthen the muscle tendon. This exercise can provide neuromuscular facilitation in both agonist and antagonist muscle [15]. 
A randomized trial by Tyler et al. [16] conducted on lateral epicondylitis. The aim of this study was to find out the effectiveness of eccentric exercise compared with standard physiotherapy treatment. The total participants were 21 . The eccentric group $(n=11)$ used eccentric wrist extensor strengthening by using flexi bar. The standard group received isotonic wrist extensor lengthening $(n=10)$. The treatment dose was 15 repetitions in 3 sets. The procedure is showed in figure 1 . The participants were suffering the symptoms of lateral epicondylitis for more than 6 weeks. Their diagnosis was confirmed by resisted wrist extension and resisted middle finger extension. All participants were received the usual care consist of wrist extensor stretching, ultrasound, cross-friction massage, heat and ice. The outcome measures were visual analogue scale (VAS), dynamometer and DASH (disability of arm, shoulder and hand) questionnaire. The both groups did not differ the outcome. For DASH score-there are statistically significant improvement in eccentric group $(p=0.01)$. The 5 patients did $>90 \%$ improvement but in standard treatment no patients had $>90 \%$ improvement in DASH score. The eccentric group showed better reduction of pain in VAS of eccentric group vs standard group (mean improvement $81 \%$ vs $22 \%$. In this group $90 \%$ pain was reduced in VAS whereas no participants had $>90 \%$ improvement. In wrist extension strength improved $(p=0.005)$ but not statistically in standard group ( $p=0.43$ ). The tenderness also reduced in eccentric group but unchanging in standard group. All outcome has improved in eccentric training group. So lastly it is concluded that eccentric exercise by using flexi bar is effective in patient with lateral epicondylitis.

Page [17] stated that eccentric exercise is a novel exercise which reduces the symptoms of lateral epicondylitis and advice to clinician for using this exercise as evidence-based intervention. This eccentric exercise provides structural and functional benefit during rehabilitation.

\section{Return to sports after rehabilitation}

Athlete will be return to work with 6 weeks after rehabilitation. Tennis elbow takes from 6 months to 12 months to heal. If a tennis player covers full rehabilitation phase, then they can return to play as early as possible. The patient can return to play at one and two months follow up by maintaining the aggravating factors. A case study by Howitt [18] conducted a case study who was diagnosed in lateral epicondylitis at right elbow. He has been suffering elbow for three months. In visual analogue scale the pain was $8 / 10$. On that time the patient was not able to play tennis. After one month he was continued tennis with the elbow strap. Lastly the patient was playing tennis 2-3 times per week. But he was advised to use a light, non-vibrational racquet, to improve the stroke technique to prevent any recurrences. In VAS he was no pain $(0 / 10)$. In that time the patient was completed the rehabilitation program. In figure 2 have the rehabilitation program.

\section{Prevention of lateral epicondylitis}

Warm up exercise is a crucial part before and after participating in any sport that can reduce the further injuries and helpful for lasting the performance. In a study it is found that athlete had done warm up the occurrence of injury was decreased. In this epidemiological study professional tennis athlete was recruited. The athlete who did not perform any warm up session they developed maximum frequency of injury. There were 4 warm up group in this study. Group $1=$ no warm up, group $2=5$ minutes, group $3=5$ to 10 minutes and group $4=$ more

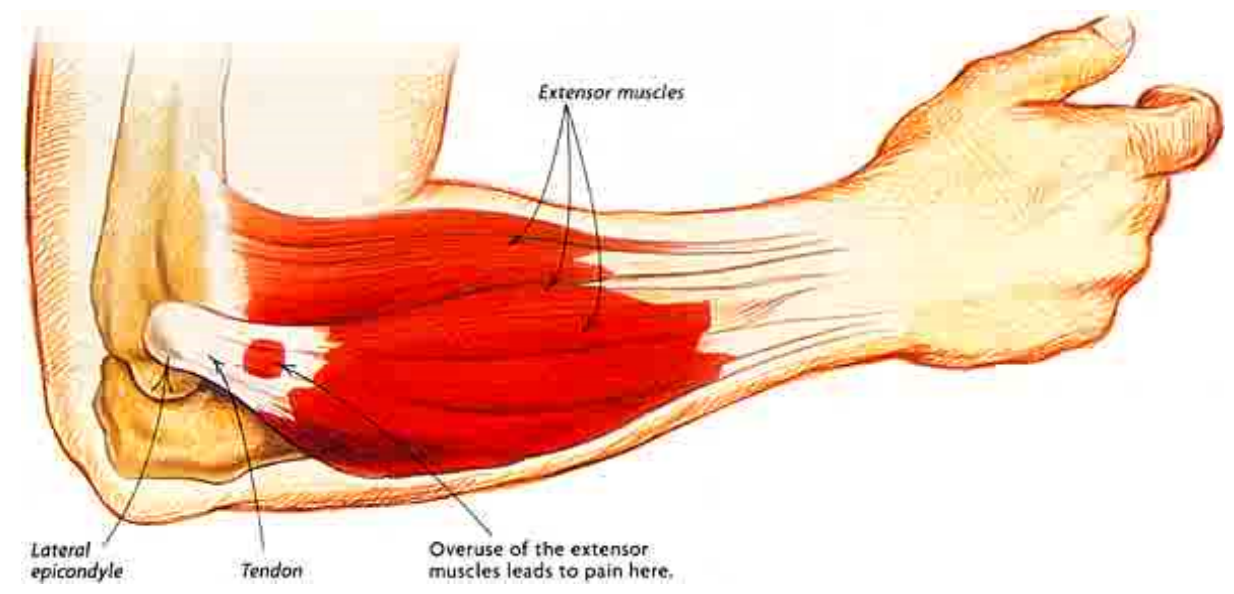

Figure 1. Showing the involvement of extensor group of muscle in lateral epicondylitis
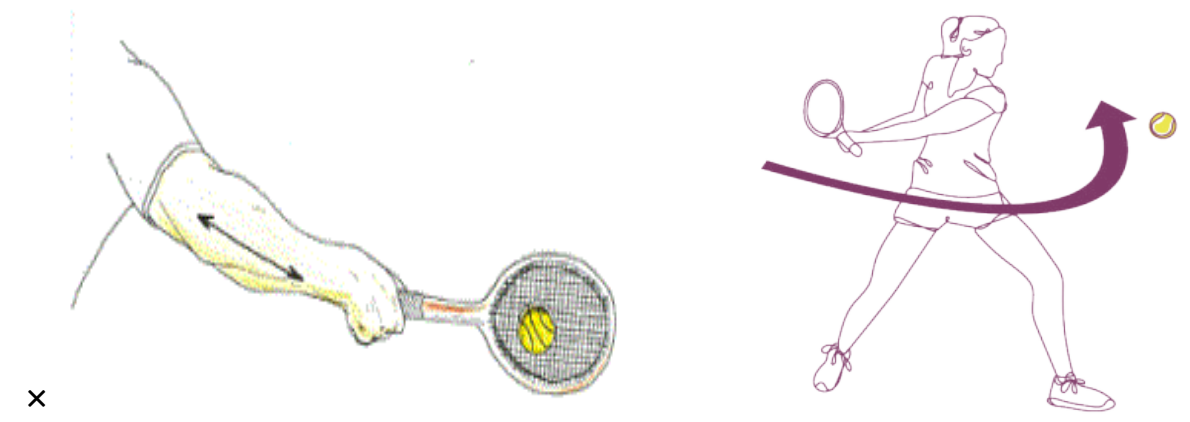

Figure 2. Showing improper and correct tennis backhand swing 
than 10 minutes warm up by participants. The incidence of injuries in Groups 1, 2, 3 and 4 was 2.65, 2.27, 2.13 and 1.54. So, it is clearly stated that warm up decreases the incidence rate of tennis elbow [8].

A tennis elbow strap or counterforce brace is effective to prevent tennis elbow. It is designed to be worn 2-3 centimeters below the elbow. The aim is planned to take the stress off the tendon. The brace is to be worn during sports or work and does not need to be worn at rest. In figure 4 demonstrated the image of brace.

A study by Ahmed [19] conducted a case control study. Total participants were 60 . The author found that majority of participants develop tennis elbow who over used the hand (more than 2 hours, heavy weight lifting by hand ( $\geq 15$ kilogram) and recurrence of injury. So lastly, he recommended that the modification of over use of hand and weight lifting for can be some preventive measures for developing tennis elbow. The modification of the working position, correction the hand during playing and the daily living activities can prevent the tennis elbow (Table 1).

A one-handed backhand performed with poor technique is a common cause of tennis elbow. The one-handed backhand places a lot of strain on the muscles and tendons of the forearm that results the pain on the outside of the elbow and back of the forearm.

So, for preventive purpose following measures should be maintained for the tennis player.

- Two handed backhand instead of a single handed backhand; turn the shoulder and trunk into the swing; and when the racket contacts the ball, the wrist should be slightly extended and deviated upward instead of flexed and deviated downward.
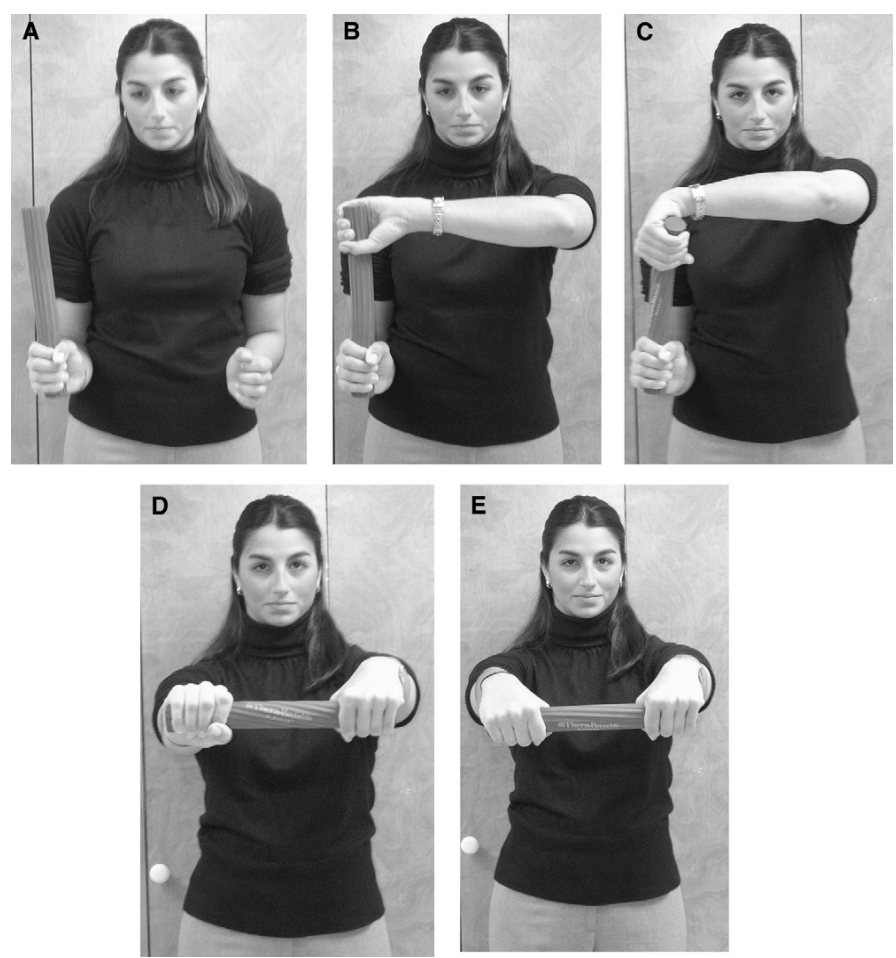

Figure 3. The procedure of eccentric exercise by using flexi bar A: Involved (right) hand in maximum wrist extension; B: The flexi bar grasped by non-involved (left) hand; C: The flexi bar twisted by flexing the non-involved wrist while holding the involved wrist in extension; D: Elbows in extension while maintaining twist in rubber bar by holding with non-involved wrist in full flexion and the involved wrist in full extension; E: The flexi bar slowly untwisted by allowing involved wrist to move into flexion

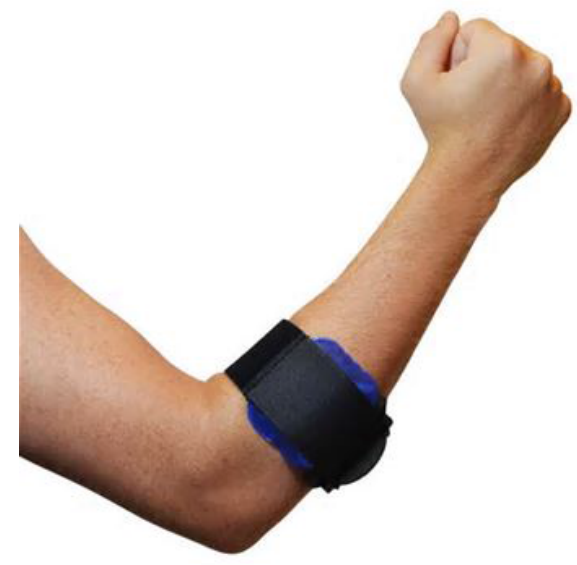

Figure 4. The counterforce brace

Table 1. The rehabilitation programs

\begin{tabular}{|l|l|}
\hline Exercise & Doses \\
\hline General Stretching - shoulder, elbow, wrist & $\begin{array}{l}\text { Active isolated stretching - dynamic } \\
5 \text { minutes warm-up }\end{array}$ \\
\hline Wrist Flexion/Extension & $\begin{array}{l}3 \text { sets of } 10 \text { with surgical tubing of } \\
\text { increasing difficulty }\end{array}$ \\
\hline Elbow Flexion/Extension & As above \\
\hline Finger Extension/Flexion & $\begin{array}{l}\text { For } 1 \text { minute with hand webs of increasing } \\
\text { difficulty }\end{array}$ \\
\hline Forearm Pronation/Supination & 3 sets of 10 with a $5 \mathrm{lb}$ weighted hammer \\
\hline Radial/Ulnar Deviation & 3 sets of 10 with a $3 \mathrm{lb}$. weighted racquet \\
\hline Eccentric loading of wrist extensors & 1 set of 10 with a $25 \mathrm{lb}$. dumbbell \\
\hline $\begin{array}{l}\text { Plyometric, purse drop (extend from full } \\
\text { flexion) }\end{array}$ & 1 set of 10 with $5 \mathrm{lbs}$ of weight \\
\hline $\begin{array}{l}\text { Simulated tennis swings }- \text { forehand, } \\
\text { backhand, and serve }\end{array}$ & $\begin{array}{l}3 \mathrm{lb} \text {. weighted tennis racquet to Cable } \\
\text { cross }-1 \text { set of } 20 \text { for each with 5-10 lbs } \\
\text { of resistance }\end{array}$ \\
\hline
\end{tabular}

- Select the proper racket, keeping in mind grip size, racket weight, and string tension.

- Be sure to properly warm up before play begins.

- After the match is over, ice the elbow.

- Emphasize strength and flexibility training for the forearm, trunk, and shoulder musculature.

- Not to grip the racquet too tightly that causes wrist less flexible in rotating and flicking.

\section{Discussion}

Lateral epicondylitis is the soft tissue injury of elbow. The patient complains pain and tenderness over lateral epicondyle of the humerus and sometimes radiate to forearm. The dominant arm is commonly affected. The aim of this study is to find out the effectiveness of isotonic strength training for lateral epicondylitis. In all included article there were used combination of treatment so that it is very difficult to conclude that one treatment technique is superior to other and found some contradictory result.

In isotonic strength training the use of concentric or eccentric strength training is contradictory. But evidence supported for the use of eccentric strength training combined with other approaches like stretching, isometric strengthening and other standard therapeutic approaches. There are three principles of eccentric training consists of load, speed and velocity of contraction. The load should be increased, 
slow the speed to avoid pain and velocity of contraction in 10 repetitions, 3 sets [20].

To find out the effectiveness of eccentric or concentric for lateral epicondylitis patient, 94 participants were divided in three groups. $1^{\text {st }}$ group have taken stretching exercise, $2^{\text {nd }}$ group used concentric strengthening and stretching, $3^{\text {rd }}$ group received eccentric strengthening and stretching. Outcome measures were VAS, DASH and Patient-rated Forearm Evaluation Questionnaire. In result there was no significant difference between 3 groups [21].

Stasinopoulos and Stasinopoulos [22] investigated that eccentricconcentric training combined with isometric contractions was better benefitted for the treatment of lateral epicondylitis. A randomized control trial was conducted in to three groups. Group A used eccentric training, group $\mathrm{B}$ received eccentric concentric training and group $\mathrm{C}$ used eccentric-concentric training with isometric contraction. In posttest and follow up test (after 1 month or 8 week) group C (eccentric-concentric training with isometric contraction) showed better improvement in function and reduction of pain $(p<0.05)$.

A case study by Dimitrios [23] found that the combination of eccentric training of wrist extensors with isometric contractions of wrist extensors and static stretching exercises of wrist extensors had improvement for reduction of pain, improvement of function and grip strength. The author said that eccentric training is the component of rehabilitation. For the management of lateral epicondylitis eccentric exercise combined with isometric contraction the outcome is higher. In this study he used 3 techniques combinedly: eccentric training, isometric contraction and static stretching of wrist extensors. The dose was 10 repetitions, 3 sets. The static stretching: elbow was placed in full extension, the forearm in full pronation, and the wrist in flexion and ulnar deviation and held for 30-45 seconds. The isometric contraction of wrist extensors for 10 seconds. The Eccentric exercises: elbow on in full extension, the forearm in pronation, the wrist in an extended position and the hand hanging over the edge of the bed. Then patient flexed the wrist slowly while counting to 30 . After $8^{\text {th }}$ week the pain in VAS, the score was $2 / 10$, pain free grip strength was improved, (from $26 \mathrm{lb}$ to $64 \mathrm{lb}$ ). The function was also measure by VAS where 0 indicate no function and 10 indicate full function. But function measured by VAS is not valid and reliable.

A RCT by Tyler et al. [16] found eccentric exercise is effective by using flexi bar that described previously.

By using 34 patients another randomized controlled trial was conducted on lateral epicondylitis. The experimental group used eccentric exercise with standard physiotherapy and control group used only standard therapy on pain and grip strength. The procedure was same as previous study. Or see the figure 1 . This study found that both groups showed statistically significant improvement, but eccentric exercise group showed better improvement in pain and grip strength. The authors suggested to use this technique in the management of lateral epicondylitis [24]. In contrast when eccentric exercise combined with electrotherapy then outcome is not satisfactory. In the study of Nagrale, [25] on lateral epicondylitis could not found that eccentric exercise can reduce pain and improve strength and function. Previously it is stated that the combination of eccentric exercise had greater improvement on pain, strength and function rather than isolated eccentric exercise.

\section{Conclusion}

Lateral epicondylitis is the frequent overuse injury of elbow. It occurs in repetitive upper limb activities in forceful forearm pronation and supination. There are different types of therapeutic treatments are available to treat lateral epicondylitis. The body of evidence provides the knowledge about the role of isotonic strength training for lateral epicondylitis. Initially it is managed by the application of ice, the use of counterforce strap and with avoiding of aggravating factors. The use of isotonic strength training especially eccentric strength training is effective to reduce pain, improvement of strength and function. To conclude it is stated that professional can use this technique as evidencebased approach to manage the patients with lateral epicondylitis.

\section{Key message}

- This present study showed that eccentric strength training of wrist extensors is effective for patients with lateral epicondylitis in reducing pain, improvement of function and strength.

- The combination of eccentric strength training with other approaches are useful on reducing pain and improving strength with function.

\section{References}

1. Physiotutors. Cozen's Test, Lateral Epicondylitis "Tennis Elbow". Available at https:// www.youtube.com/watch? $=8 \mathrm{~K} 7 \mathrm{jzDIUpLI}$

2. Longo UG, Franceschetti E, Rizzello G, Petrillo S, Denaro V (2012) Elbow tendinopathy. Muscles Ligaments Tendons J 2: 115-120. [Crossref]

3. Tajika T, Kobayashi T, Yamamoto A, Kaneko T, Takagishi K (2014) Prevalence and risk factors of lateral epicondylitis in a mountain village in Japan. J Orthop Surg (Hong Kong) 22: 240-243 [Crossref]

4. Shiri R, Viikari-Juntura E, Varonen H, Heliövaara M (2006) Prevalence and Determinants of Lateral and Medial Epicondylitis: A Population Study. Am J Epidemiol 164: 1065-1074. [Crossref]

5. Sanders TL Jr, Maradit Kremers H, Bryan AJ, Ransom JE, Smith J (2015) The epidemiology and healthcare burden of tennis elbow: a population-based study. $\mathrm{Am} \mathrm{J}$ Sports Med 43: 1066-1071. [Crossref]

6. Alizadehkhaiyat O, Fisher AC, Kemp GJ, Vishwanathan K, Frostick SP (2007) Upper Limb Muscle Imbalance in Tennis Elbow: A Functional and Electromyographic Assessment. J Orthop Res 25: 1651-1657. [Crossref]

7. Kolo, (2018) Tennis elbow. Encyclopaedia Britanica, [Online], Available at: https:// www.britannica.com/science/tennis-elbow

8. Kachanathu SJ, Kumar P, Malhotra M (2014) Relevance and Incidence of Musculoskeletal Injuries in Indian Tennis Players; an Epidemiological Study. American Journal of Sports Science and Medicine 2: 1-5.

9. Lucado AM, Kolber MJ, Cheng MS, Echternach JL (2012) Upper Extremity Strength Characteristics in Female Recreational Tennis Players with and Without Lateral Epicondylalgia. J Orthop Sports Phys Ther 42: 1025-1031. [Crossref]

10. Taylor SA, Hannafin JA (2012) Evaluation and management of elbow tendinopathy. Sports Health 4: 384-393. [Crossref]

11. Sims SE, Miller K, Elfar JC, Hammert WC (2014) Non-surgical treatment of latera epicondylitis: A Systematic Review of Randomized Controlled Trials. Hand 9: 419446. [Crossref]

12. Rana J, Rakib (2017) Interview on Tennis Elbow Interviewed by Rabea Begum [Face to Face interview] Dhaka, 21st September 2017, 5pm.

13. Cullinane FL, Boocock MG, Trevelyan FC (2014) Is eccentric exercise an effective treatment for lateral epicondylitis? A systematic review. Clin Rehabil 28: 3-19. [Crossref]

14. Mac Auley DC (2001) Ice therapy: how good is the evidence? Int J Sports Med 22 379-384. [Crossref]

15. Pensini M, Martin A, Maffiuletti N (2002) Central versus peripheral adaptations following eccentric resistance training. Int J Sports Med 23: 567-574. [Crossref]

16. Tyler TF, Thomas GC, Nicholas SJ, McHugh MP (2010) Addition of isolated wrist extensor eccentric exercise to standard treatment for chronic lateral epicondylosis: A prospective randomized trial. J Shoulder Elbow Surg 19: 917-922. [Crossref] 
17. Page P (2010) A new exercise for tennis elbow that works! N Am J Sports Phys Ther 5: 189-193 [Crossref]

18. Howiit SD (2006) Lateral epicondylosis: a case study of conservative care utilizing ART and rehabilitation. J Can Chiropr Assoc 50: 182-189. [Crossref]

19. Ahmed S (2013) Risk factors of tennis elbow patients attended at two selected organizations in Dhaka. [Online] Available at: http://library.crp-bangladesh.org:8080/ xmlui/handle/123456789/72

20. Stanish WD, Rubinovich RM, Curwin S (1986) Eccentric exercise in chronic tendinitis. Clin Orthop Relat Res 208: 65-68. [Crossref]

21. Martinez-Silvestrini JA, Newcomer KL, Gay RE, Schaefer MP, Kortebein P, et al. (2005) Chronic Lateral Epicondylitis: Comparative Effectiveness of a Home Exercise Program Including Stretching Alone versus Stretching Supplemented with Eccentric or Concentric Strengthening. J Hand Ther 18: 411-419 [Crossref]
22. Stasinopoulos D, Stasinopoulos I (2017) Comparison of effects of eccentric training, eccentric-concentric training, and eccentric-concentric training combined with isometric contraction in the treatment of lateral elbow tendinopathy. J Hand Ther 30: 13-19. [Crossref]

23. Dimitrios S (2015) The Effectiveness of Isometric Contractions Combined with Eccentric Contractions and Stretching Exercises on Pain and Disability in Latera Elbow Tendinopathy. A Case Report. J Nov Physiother 5: 2-5.

24. Majeedkutty NA, Majida NAL (2016) Effects of Therapeutic Eccentric Exercise on Pain and Grip Strength in Persons with Lateral Epicondylitis: A Randomized Controlled Trial. IOSR-JNHS 5: 66-71.

25. Amit V Nagrale, Christopher R Herd, Shyam Ganvir, Gopichand Ramteke (2009) Cyriax Physiotherapy Versus Phonophoresis with Supervised Exercise in Subjects with Lateral Epicondylalgia: A Randomized Clinical Trial J Man Manip Ther 17: 171-178[Crossref]

Copyright: ( 2019 Begum R. This is an open-access article distributed under the terms of the Creative Commons Attribution License, which permits unrestricted use, distribution, and reproduction in any medium, provided the original author and source are credited. 